PAPER

\title{
The beneficial antispasticity effect of botulinum toxin type $A$ is maintained after repeated treatment cycles
}

\author{
A M O Bakheit, N V Fedorova, A A Skoromets, S L Timerbaeva, B B Bhakta, L Coxon
}

J Neurol Neurosurg Psychiatry 2004;75:1558-1561. doi: 10.1136/jnnp.2003.035139

See end of article for authors' affiliations

Correspondence to:

Professor A M O Bakheit, Mount Gould Hospital,

Plymouth PL4 7QD, UK magid.bakheit@ pcs-tr.swest.nhs.uk

Received

29 December 2003

In revised form

14 February 2004

Accepted

15 February 2004

\begin{abstract}
Objective: To study the efficacy, safety, and incidence of BtxA antibody formation with repeated treatments with BtxA in post-stroke upper limb muscle spasticity.

Methods: The study was a prospective open label trial. Patients with established post-stroke upper limb spasticity received 1000 units of BtxA (Dysport) into five muscles of the affected arm on study entry. Treatment was repeated every 12, 16, or 20 weeks as clinically indicated. Each patient received a total of three treatment cycles. Efficacy of treatment was assessed using the Modified Ashworth Scale. Patients were assessed on study entry and on week 4 and 12 of each treatment cycle for all safety and efficacy parameters. Blood samples for BtxA antibody assay were taken at baseline and on completion of the trial. Results: Fifty one patients were recruited and 41 of them completed the study. Improvement from the cycle one baseline was observed in all the outcome measures. Mild to moderately severe treatment related adverse events were reported in $24 \%$ of cases. There were no serious adverse events. No BtxA antibodies were detected.

Conclusion: BtxA at a dose of 1000 units Dysport was efficacious in the symptomatic treatment of poststroke upper limb spasticity. The study suggests that this effect can be maintained with repeated injections for up to at least three treatment cycles, with duration of effect per cycle of between 12 and 20 weeks. BtxA was safe in the dose used in this study and did not induce the formation of detectable levels of neutralising BtxA antibodies.
\end{abstract}

$\mathrm{S}$ evere, disabling spasticity of upper limb muscles is a common complication in patients with stroke. It frequently causes difficulties with activities of daily living, such as dressing and cleaning the palm of the clenched hand. It may also interfere with voluntary motor function in patients with residual muscle strength. ${ }^{1}$ In some patients spasticity also causes persistent pain or discomfort. Treatment of spasticity with the traditional muscle relaxant drugs often results in systemic adverse effects. It may also lead to loss of motor function-for example, the patient may become unable to maintain the sitting posture because of the weakening of the trunk muscles. Furthermore, the beneficial effect of the systemic antispasticity agents may diminish with prolonged use as tolerance to these drugs usually develops after a few months of treatment.

In recent years, large randomised controlled trials (RCTs) have shown botulinum toxin type A (BtxA) to be safe and efficacious in the symptomatic relief of muscle spasticity due to stroke, ${ }^{2-5}$ multiple sclerosis, ${ }^{6}$ and spastic cerebral palsy. However, all previous studies evaluated the safety and efficacy of a single treatment cycle. The function of the neuromuscular junction is usually fully restored within 3-4 months after the BtxA injection. ${ }^{8}$ Consequently, in most patients, treatment with BtxA often needs to be repeated three or four times a year, possibly for many years. However, at present there is no research evidence on whether the beneficial effect of BtxA on muscle tone is maintained with repeated treatment cycles.

One problem associated with repeated treatment can be a failure to benefit from the treatment long term. This has been reported following repeated BtxA injections for cervical dystonia, ${ }^{9}$ blepharospasm, and hemifacial spasm. $^{10}$ This secondary treatment failure was found to be because of immunological resistance resulting from BtxA antibody formation and was attributed to a large cumulative dose of the toxin. The dose of BtxA that is normally used in the treatment of muscle spasticity is significantly larger than the amount required for blepharospasm, hemifacial spasm, and cervical dystonia. It is therefore important to establish whether immunological resistance occurs after repeated use of BtxA in the relatively large doses that are required to relieve muscle spasticity.

The aim of the present study was to investigate the efficacy and safety of repeated treatment cycles with BtxA (Dysport, Ipsen Ltd, UK) in post-stroke upper limb spasticity and to determine the incidence of neutralising BtxA antibodies in these patients.

\section{METHODS}

\section{Study design and patient recruitment}

This was an international, multicentre $(n=5)$, open label study. Approval by the appropriate ethics committees and patients' written informed consent to participate in the study were obtained before starting the trial.

Adult patients with hemiplegic stroke and severe or moderately severe upper limb muscle spasticity were recruited at least 3 months after the onset of the cerebrovascular event. They were included in the study if they had a muscle tone score of two or more on the Modified Ashworth Scale $(\mathrm{MAS})^{11}$ in moving at least two joints of the elbow, wrist, and fingers, and a score of $1+$ in the remaining area. In addition, patients were recruited only if they had a minimum score of 10 in the Patients' Disability and Carer Burden Rating scale (PD \& CBRS). ${ }^{4}$

Patients with fixed muscle contractures of the upper limb were excluded. (A fixed muscle contracture in this study was defined as severe restriction of the joint range of motion on passive muscle stretch.) Other exclusion criteria were previous treatment with phenol or alcohol nerve blocks or motor point injections for upper limb spasticity at any time

Abbreviations: BtxA, botulinum toxin type A; MAS, Modified Ashworth Scale; PD \& CBRS, Patients' Disability and Carer Burden Rating scale; $R C T$, randomised controlled trials; ROM, range of motion. 
and treatment with BtxA in the 90 days preceding the study or treatment with intrathecal baclofen. Stroke was defined according to the World Health Organization criteria. ${ }^{12}$ Assessment of the patients' motor, sensory, and perceptual function was made with standard clinical examination.

Patients were screened for their eligibility for the trial and those who met the study entry criteria were re-assessed 4 weeks later. If there had been no change in their MAS scores, and if their baseline PD \& CBRS scores remained unchanged or decreased by no more than five points, they were enrolled into the trial and received their first cycle of treatment. The continued use of physiotherapy, occupational therapy, orthoses, and antispasticity medication-for example, oral baclofen-was permitted if these treatments had been started before entry into the study and they remained unchanged until completion of the trial.

\section{Treatment}

BtxA (Dysport, Ipsen Ltd, UK) was presented as a freezedried pellet of 500 units of botulinum A toxin-haemagglutinin complex, $125 \mu \mathrm{g}$ of human albumin, and $2.5 \mathrm{mg}$ of lactose. The contents of the vial were reconstituted in $1 \mathrm{ml}$ of $0.9 \%$ sodium chloride solution. A total of 300-400 units of BtxA were injected into the biceps brachii, 150-250 units into the flexor digitorum superficialis, and 150 units into each of the flexor digitorum profundus, flexor carpi ulnaris, and flexor carpi radialis. Only one site per muscle was injected except for the biceps brachii, which was injected in two sites. The injections were placed in the muscle belly close to the motor endplate zone using anatomical landmarks as in routine electromyography..$^{13}$ A total of 1000 units of BtxA was injected in the first treatment cycle. In cycles 2 and 3 the same muscles were injected but the dose was adjusted at the discretion of the clinician and ranged from 500-1000 units according to the patients' clinical needs.

Treatment cycles were separated by either 12 or 16 weeks. Patients were re-injected on week 12 if the MAS spasticity score had returned to its baseline value. However, if at week 12 the hypertonis was still reduced by one grade or more at the elbow, wrist, or fingers, the injections were deferred until week 16.

\section{Assessments}

Patients were assessed, by an independent assessor, on study entry and at the end of week 4 of each treatment cycle for safety and efficacy measures and at week 12 for retreatment. The final assessments were made 12 weeks after the third treatment cycle. The efficacy of treatment was determined by comparing the scores of the outcome measures at week 4 of each treatment cycle with those of cycle 1 baseline assessment.

\section{Outcome measures}

Efficacy of treatment was measured with the following assessment scales:

1. The MAS: ${ }^{10}$ muscle tone was assessed separately at the elbow, wrist, and fingers. The degree of resistance to the passive muscle stretch that was felt by the examiner was scored on a six point scale ranging from 0 (no increase in muscle tone) to 4 (the affected part is rigid in flexion and extension).

2. The joint range of motion (ROM) on voluntary extension of the elbow and wrist and on passive muscle stretch was measured using a hand-held goniometer. The ROM was calculated as the maximum angle of flexion minus the maximum angle of extension. Finger flexion was assessed for active and passive maximum extension of the hand. The joint ROM in the fingers is difficult to measure accurately with goniometry. It was therefore assessed according to the following scale: hand closed, quarter open, half open, three quarters open, or fully open with active movement or passive muscle stretch. A decrease in the ability to open the hand was recorded as a negative number and an increase as a positive number-for example, a change from "hand closed" to "quarter open" received the score of 1 whereas the opposite was scored as -1 .

3. The severity of muscle pain at the shoulder, arm, wrist, and fingers at rest or on movement was assessed on a four point scale $(0=$ no pain, $1=$ mild, $2=$ moderate, $3=$ severe).

4. Goal attainment scale: the investigator discussed the rationale for treatment of muscle spasticity with each patient and identified and agreed with him/her three realistic functional goals prior to the injection. Attainment of the agreed goals was recorded at each follow up visit as "goals achieved" or "goals not achieved".

5. PD \& $\mathrm{CBRS}^{4}$ the first part of this scale (patient disability) consists of eight items (cleaning the palm of the hand, cutting fingernails, putting the paretic arm through a sleeve, cleaning under the armpit, cleaning around the elbow, standing balance, walking balance, and the ability to perform home arm physiotherapy). The second part (carer burden) consists of four items (cleaning the palm, cutting fingernails, dressing, and cleaning under the armpit). Each category is graded as follows: $0=$ no disability/carer burden; $1=$ mild disability/carer burden; $2=$ moderate disability/carer burden; $3=$ severe disability/carer burden; $4=$ maximum disability/carer burden. The scores are added up to give the total scores for the PD \& CBRS. A score of 32 means that the patient is unable to perform any of the tasks, whereas a score of zero means the opposite. To standardise the scores across patients performing different numbers of activities, the obtained score was divided by the number of activities performed within the scale. Changes were expressed in terms of this value.

6. Global assessment of benefit: at the end of the study the patients and investigators were asked to give an overall subjective assessment of the treatment effect according to one of the following descriptions: great benefit, some benefit, symptoms unchanged, symptoms worse, or symptoms much worse.

7. Request for future treatment: patients were asked on completion of cycle 3 if they wished to receive BtxA in the future if their symptoms recurred. The investigators were also asked if they would recommend further injections for their patients.

Safety was assessed by asking the patients to describe any adverse events that they had experienced since the previous visit. In addition, a clinical examination was conducted by the investigators at each clinic visit.

\section{Botulinum toxin antibody assay}

Venous blood samples $(10 \mathrm{ml})$ were collected at baseline and on completion of the third treatment cycle. BtxA antibodies were measured by Wickham Laboratories Ltd, Fareham, UK, using the standard mouse lethality assay $\left(\mathrm{LD}_{50}\right) .{ }^{14}$ The results of BtxA antibody titres were reported as nil, low, low/ intermediate, intermediate, intermediate/high, or high.

\section{RESULTS}

A total of 55 patients were screened and 51 of them fulfilled the study criteria and were recruited. The patients' demographic and clinical characteristics are given in table 1. Forty 
Table 1 The demographic and clinical characteristics of the study population $(n=51)$

\begin{tabular}{lc}
\hline Mean age in years (SD) & $56.2(11.5)$ \\
Sex & $30(59 \%)$ \\
Male & $21(41 \%)$ \\
Female & $29(57 \%)$ \\
Ischaemic stroke & $6(11 \%)$ \\
Haemorrhagic stroke & $4(8 \%)$ \\
Cerebral embolism & $1(2 \%)$ \\
Subarachnoid haemorrhage & $11(22 \%)$ \\
Not known & $18(35 \%)$ \\
Hemiparetic arm & $33(65 \%)$ \\
$\quad$ Dominant & $27(53 \%)$ \\
$\quad$ None dominant & $28(55 \%)$ \\
Unilateral hemispatial neglect present \\
Disturbances of upper limb sensation \\
present
\end{tabular}

patients completed the first treatment cycle, 43 completed cycle 2, and 41 completed cycle three. Eight patients withdrew their consent during the course of the study, one patient died from a cerebro-vascular event, and one patient was lost to follow up. No patient withdrew from the study because of adverse events. The data were analysed for efficacy on an intention to treat basis. Five patients were receiving oral baclofen and 22 were receiving physiotherapy and/or occupational therapy on study entry. These treatments were continued unchanged during the study period.

\section{Efficacy}

As shown in tables 2-5, BtxA in the dose used in this study reduced muscle tone in the spastic upper limb as well as the patient's disability and the carer's burden of nursing care. The identified treatment goals were achieved by more than half the study patients. Furthermore, the range of active and passive movement at the upper limb joints showed a modest increase in the elbow and wrist and there was a reduction in muscle pain in some patients. These improvements were maintained throughout the three treatment cycles.

The global assessment found that thirty seven (90\%) of the 41 patients who completed the final assessment reported that treatment was beneficial overall and 31 (76\%) of them requested future injections with BtxA. However, the investigators recorded that treatment was beneficial in 39 (95\%) of patients and recommended that BtxA injections should be considered in the future for $31(76 \%)$ of them.

Table 2 Summary of the number and percentage of patients with improvements in four study variables from cycle 1 baseline to week 4 of each cycle (intention to treat population)

\begin{tabular}{|c|c|c|c|}
\hline Variable & $\begin{array}{l}\text { Cycle } 1 \\
n=51\end{array}$ & $\begin{array}{l}\text { Cycle } 2 \\
n=46\end{array}$ & $\begin{array}{l}\text { Cycle } 3 \\
n=42\end{array}$ \\
\hline $\begin{array}{l}\text { Decrease of } 1 \text { on MAS in } \\
\text { at least one joint }\end{array}$ & $50(100 \%)$ & $44(98 \%)$ & $39(98 \%)$ \\
\hline $\begin{array}{l}95 \% \text { confidence interval } \\
\text { (CI) }\end{array}$ & $(93 \%, 100 \%)$ & $(88 \%, 100 \%)$ & $(87 \%, 100 \%)$ \\
\hline $\begin{array}{l}\text { Mean (SD) decrease in } \\
\text { Patients' Disability Rating } \\
\text { scale }\end{array}$ & $0.31(0.32)$ & $0.47(0.42)$ & $0.54(0.55)$ \\
\hline $95 \% \mathrm{Cl}$ & $(0.40,0.22)$ & $(0.60,0.34)$ & $(0.71,0.36)$ \\
\hline $\begin{array}{l}\text { Mean (SD) decrease in } \\
\text { Carers Burden scale }\end{array}$ & $0.34(0.49)$ & $0.33(0.49)$ & $0.27(0.44)$ \\
\hline $95 \% \mathrm{Cl}^{*}$ & $(0.49,0.18)$ & $(0.50,0.15)$ & $(0.45,0.08)$ \\
\hline Goals achieved & $26(52 \%)$ & $25(56 \%)$ & $23(58 \%)$ \\
\hline
\end{tabular}

*Responses were received from 40 carers in cycle one, from 33 in cycle 2, and 24 from cycle 3. MAS, Modified Ashworth Scale.
Table 3 Summary of the change in range of active movement of the elbow, wrist, and fingers from cycle 1 baseline to week 4 of each treatment cycle (intention to treat population)

\begin{tabular}{|c|c|c|c|}
\hline $\begin{array}{l}\text { Change from cycle } 1 \\
\text { baseline to week } 4 \\
\text { of each cycle }\end{array}$ & $\begin{array}{l}\text { Cycle } 1 \\
n=51\end{array}$ & $\begin{array}{l}\text { Cycle } 2 \\
n=46\end{array}$ & $\begin{array}{l}\text { Cycle } 3 \\
n=42\end{array}$ \\
\hline \multicolumn{4}{|l|}{ Elbow } \\
\hline $\begin{array}{l}\text { Mean (degrees [SD]) } \\
95 \% \mathrm{Cl}\end{array}$ & $\begin{array}{l}10.3(24.0) \\
(3.4,17.1)\end{array}$ & $\begin{array}{l}8.8(22.1) \\
(2.1,15.4)\end{array}$ & $\begin{array}{l}8.0(27.9) \\
(-0.9,16.9)\end{array}$ \\
\hline \multicolumn{4}{|l|}{ Wrist } \\
\hline $\begin{array}{l}\text { Mean (degrees [SD]) } \\
95 \% \mathrm{Cl}\end{array}$ & $\begin{array}{l}5.2(25.9) \\
(-2.2,12.5)\end{array}$ & $\begin{array}{l}9.6(22.0) \\
(2.9,16.2)\end{array}$ & $\begin{array}{l}11.4(20.6) \\
(4.8,18.0)\end{array}$ \\
\hline \multicolumn{4}{|l|}{ Fingers } \\
\hline Decrease $\geqslant 1$ (n [\%]) & $1(2 \%)$ & $0(0 \%)$ & $0(0 \%)$ \\
\hline No change (n [\%]) & $25(50 \%)$ & $18(40 \%)$ & $14(35 \%)$ \\
\hline Increase $\geqslant 1$ (n [\%]) & $24(48 \%)$ & $27(60 \%)$ & $26(65 \%)$ \\
\hline
\end{tabular}

\section{Safety}

Adverse events that were considered by the investigators to be because of BtxA were reported in $24 \%$ of cases. All the reported adverse effects were rated as mild or moderate in intensity. No fatal, life threatening, or incapacitating adverse events relating to the study medication were reported or observed. The most frequently reported unwanted treatment

Table 4 Summary of the change in range of passive movement of the elbow, wrist, and fingers from cycle 1 baseline to week 4 of each treatment cycle (intention to treat population)

\begin{tabular}{|c|c|c|c|}
\hline $\begin{array}{l}\text { Change from cycle } 1 \\
\text { baseline to week } 4 \text { of } \\
\text { each cycle }\end{array}$ & $\begin{array}{l}\text { Cycle } 1 \\
n=51\end{array}$ & $\begin{array}{l}\text { Cycle } 2 \\
n=46\end{array}$ & $\begin{array}{l}\text { Cycle } 3 \\
n=42\end{array}$ \\
\hline \multicolumn{4}{|l|}{ Elbow } \\
\hline $\begin{array}{l}\text { Mean (degrees [SD]) } \\
95 \% \mathrm{Cl}\end{array}$ & $\begin{array}{r}7.9(14.4) \\
(3.8,12.0)\end{array}$ & $\begin{array}{r}7.9(17.2) \\
(2.7,13.1)\end{array}$ & $\begin{array}{r}5.6(24.9) \\
(-23.136)\end{array}$ \\
\hline \multicolumn{4}{|l|}{ Wrist } \\
\hline $\begin{array}{l}\text { Mean (degrees [SD]) } \\
95 \% \mathrm{Cl}\end{array}$ & $\begin{array}{r}31.2(29.3) \\
(22.8,39.7)\end{array}$ & $\begin{array}{r}27.2(31.0) \\
(17.9,36.5)\end{array}$ & $\begin{array}{r}28.9(32.6) \\
(18.5,39.3)\end{array}$ \\
\hline \multicolumn{4}{|l|}{ Fingers } \\
\hline Decrease $\geqslant 1$ (n [\%]) & $0(0 \%)$ & $1(2 \%)$ & $0(0 \%)$ \\
\hline No change (n [\%]) & $37(74 \%)$ & $33(73 \%)$ & $30(75 \%)$ \\
\hline Increase $\geqslant 1(\mathrm{n}[\%])$ & $13(26 \%)$ & $11(24 \%)$ & $10(24 \%)$ \\
\hline
\end{tabular}

Table 5 Summary of the change from cycle 1 baseline to week 4 of each cycle in pain assessment (intention to treat population)

\begin{tabular}{|c|c|c|c|}
\hline Pain assessment & $\begin{array}{l}\text { Cycle } 1 \\
n=51\end{array}$ & $\begin{array}{l}\text { Cycle } 2 \\
n=46\end{array}$ & $\begin{array}{l}\text { Cycle } 3 \\
n=42\end{array}$ \\
\hline \multicolumn{4}{|l|}{ Shoulder } \\
\hline Improvement (n [\%]) & $\begin{array}{l}16(32 \%) \\
(21 \%, 46 \%)\end{array}$ & $\begin{array}{l}18(40 \%) \\
(27 \% 55 \%)\end{array}$ & $\begin{array}{l}16(40 \%) \\
(26 \% 55 \%\end{array}$ \\
\hline No improvement (n [\%]) & $34(68 \%)$ & $27(60 \%)$ & $24(60 \%)$ \\
\hline \multicolumn{4}{|l|}{ Arm } \\
\hline Improvement (n [\%]) & $10(20 \%)$ & $10(22 \%)$ & $8(20 \%)$ \\
\hline $95 \% \mathrm{Cl}$ & 3\%) & $(13 \%$ & $(10 \%, 35 \%)$ \\
\hline No improvement (n [\%]) & $40(80 \%)$ & $35(78 \%)$ & $32(80 \%)$ \\
\hline \multicolumn{4}{|l|}{ Wrist } \\
\hline Improvement (n [\%]) & $11(22 \%)$ & $9(20 \%)$ & $5(13 \%)$ \\
\hline $95 \% \mathrm{Cl}$ & $(13 \%, 35 \%)$ & $(11 \%, 34 \%)$ & $(5 \%, 26 \%)$ \\
\hline No improvement (n [\%]) & $39(78 \%)$ & $36(80 \%)$ & $35(88 \%)$ \\
\hline \multicolumn{4}{|l|}{ Fingers } \\
\hline Improvement (n [\%]) & $12(24 \%)$ & $11(24 \%)$ & $7(18 \%)$ \\
\hline $95 \% \mathrm{Cl}$ & & $(14 \%, 39 \%)$ & $(9 \%, 32 \%)$ \\
\hline No improvement (n [\%]) & $38(76 \%)$ & $34(76 \%)$ & $33(83 \%)$ \\
\hline
\end{tabular}


effects were pain at the injection site (24\%), fatigue and tiredness (14\%), and dysphagia (2\%).

\section{Botulinum toxin antibodies}

Blood samples were obtained from 47 patients at baseline and from 32 at the final assessment. BtxA neutralising antibodies were not detected in any of the patients.

\section{DISCUSSION}

The present study has demonstrated that treatment with BtxA reduces post-stroke upper limb muscle spasticity and its sequelae and supports the findings of previous RCTs. ${ }^{2-6}$ In addition, it also provides preliminary evidence that the beneficial effect of BtxA can be maintained with repeated injections without inducing BtxA antibody formation for up to at least three consecutive treatment cycles.

Some methodological aspects of this study deserve further comments. The study protocol required a 4 week lapse after the patients' initial screening before a further assessment and the first cycle treatment are carried out. The purpose of this strategy was to allow patients enough time to consider their willingness to participate in the study and to ensure that the muscle hypertonia and its effects on motor function remain unchanged. We also chose week 4 as the optimal time point for the assessment of treatment efficacy because the clinical effect of BtxA in reducing spasticity peaks around this time. ${ }^{15}$ The study design was open label and was chosen because a placebo controlled trial long term is difficult to justify on ethical grounds.

Although open label trials are liable to bias in the measurement of the treatment effect, we believe that at least two factors argue against a major influence of this study design on our reported results. First, the results of our study are concordant with those of previously published large RCTs that evaluated the antispasticity effect of BtxA over one treatment cycle. Secondly, our own empirical observations and those of others ${ }^{16}$ are in keeping with the present findings that the beneficial antispasticity effect of BtxA can be maintained with repeated treatments. Nonetheless, it would be important to seek further evidence to support our observations using more robust research methods because of a possible significant placebo effect. ${ }^{5}$ This could ideally be done with RCTs. However, it is difficult to justify the use of placebo controls for such a long period ${ }^{17}$ as required in our study or future similar trials, especially that the effectiveness of BtxA has been confirmed previously with the same research methodology, albeit for one treatment cycle. An alternative approach would be to evaluate the long term benefit using "equivalence trials". ${ }^{18}$ For example, as repeated peripheral nerve blocks have been shown to be effective in the treatment of muscle spasticity, ${ }^{19}$ a single blind comparison of repeated BtxA treatment cycles with this treatment would be ethically and scientifically acceptable.

In contrast to previous studies, ${ }^{910}$ neutralising BtxA antibodies were not detected in any of our patients. BtxA antibodies were reported in 3\% of patients with cervical dystonia who received continuous treatment for more than 2 years. ${ }^{9}$ The absence of antibodies in our patients may be because of the relatively short duration of the present study. Another possible explanation is the relatively low sensitivity of the mouse lethality assay compared to other tests, such as the immunoprecipitation assay. ${ }^{20}$ Future studies should evaluate the potential of BtxA to induce antibody formation after longer periods of treatment and with tests that are more sensitive than the mouse lethality assay

\section{ACKNOWLEDGEMENTS}

The study was sponsored by Ipsen Limited, Slough, Berkshire, UK.

\section{Authors' affiliations}

A M O Bakheit, Professor of Neurological Rehabilitation, Peninsula Medical School \& Plymouth Primary Care Trust, Mount Gould Hospital, Plymouth PL4 7QD, UK

N V Fedorova, Professor, Russian Medical Academy for Advanced Medical Studies, 5 Vtoroj Botinsky Projezd, Moscow 125101, Russia

A A Skoromets, Professor, Pavlov's State Medical University, 6/8 L. Tolstoy street, St Petersburg 197022, Russia

S L Timerbaeva, Lead Researcher, Scientific Research Institute of Neurology, Russian Academy of Medical Sciences, 80 Volokolamskoye Shosse, Moscow 123367, Russia

B B Bhakta, Senior Lecturer in Rehabilitation Medicine, University of Leeds \& St James's Hospital, Beckett St, Leeds LS9 7TF, UK

L Coxon, Clinical Project Manager, Ipsen Ltd, 190 Bath Rd, Slough

SL1 3XE, UK

Competing interests: two of the authors (AMOB \& BBB) have received in the past sponsorship for educational activities, including attendance at international scientific meetings, from Ipsen Ltd. LC is currently an employee of Ipsen Ltd.

\section{REFERENCES}

1 Mizrahi EM, Angel RW. Impairment of voluntary movement by spasticity. Ann Neurol 1979;5:494-5

2 Simpson DM, Alexander DN, O'Brien CF, et al. Botulinum toxin type $A$ in the treatment of upper extremity spasticity: a randomized, double-blind, placebocontrolled trial. Neurology 1996;46:1306-10.

3 Bakheit AMO, Thilmann AF, Ward AB, et al. A randomized, double-blind, placebo-controlled, dose-ranging study to compare the efficacy and safety of three doses of botulinum toxin type A (Dysport) with placebo in upper limb spasticity after stroke. Stroke 2000;31:2402-6.

4 Bhakta BB, Cozens JA, Chamberlain MA, et al. Impact of botulinum toxin type A on disability and carer burden due to arm spasticity after stroke: a randomised double blind placebo controlled trial. I Neurol Neurosurg Psychiat 2000;69:217-21.

5 Brashear A, Gordon MF, Elovic E, et al. Intramuscular injection of botulinum toxin for the treatment of wrist and finger spasticity after stroke. N Engl J Med 2002; 347:395-400

6 Hyman N, Barnes M, Bhakta B, et al. Botulinum toxin (Dysport) treatment of hip adductor spasticity in multiple sclerosis: a prospective, randomised, double blind, placebo controlled, dose ranging study. J Neurol Neurosurg Psychiat 2000;68:707-12.

7 Koman AL, Mooney JF 3rd, Smith BP, et al. Botulinum toxin type A neuromuscular blockade in the treatment of lower extremity spasticity in cerebral palsy: a randomised, double-blind, placebo-controlled trial. J Pediatr Orthop 2000;20:108-15.

8 De Paiva A, Meunier FA, Molgo J, et al. Functional repair of motor endplates after botulinum neurotoxin type A poisoning: biphasic switch of synaptic activity between nerve sprouts and their parent terminals. Proc Natl Acad Sci USA 1999;96:3200-5.

9 Zuber M, Sebald M, Bathien N, et al. Botulinum toxin antibodies in dystonic patients treated with type $A$ botulinum toxin: frequency and significance. Neurology 1993;43:1715-18.

10 Siatkowski RM, Tyutyunikov A, Biglan AW, et al. Serum antibody production to botulinum A toxin. Ophthalmology 1993;43:1715-18.

11 Bohannon RW, Smith MB. Inter-rater reliability of a modified Ashworth Scale of muscle spasticity. Phys Therapy 1987;67:206-7.

12 Aho K, Harmsen P, Hatano S, et al. Cerebrovascular disease in the community: results of a WHO collaborative study. Bulletin of the World Health Organisation 1980;58:113-30.

13 Delagi EF, Perotto A, lazzetti J, et al. Anatomic guide for the electromyographer. Second ed. Springfield, IL: Charles C Thomas Publishers, 1980.

14 Hatheway CH, Dang C. Immunogenicity of the neurotoxins of Clostridium botulinum. In: Jankovic J, Hallett M, eds. Therapy with botulinum toxin. New York: Marcel Dekker, 1994:93-107.

15 Therapeutic and Technology Assessment Subcommittee of the American Academy of Medicine. The clinical usefulness of botulinum toxin $A$ in treating neurologic disorders. Neurology 1990;40:1332-6.

16 Berweck S, Graham HK, Heinen F. Spasticity in children. In: Moore AP, Naumann M, eds. Handbook of botulinum toxin treatment. Second ed. Oxford, UK: Blackwell Science, 2003:297.

17 International ethical guidelines for biomedical research involving human subjects. Council for International Organisations of Medical Sciences. Geneva, 2002.

18 Emanuel EJ, Miller FG. The ethics of placebo-controlled trials - a middle ground. N Eng J Med 2001;345:915-19.

19 Bakheit AMO, Badwan DAH, McLellan DL. The effectiveness of chemical neurolysis in the treatment of lower limb muscle spasticity. Clin Rehabil 1996;10:40-3.

20 Hanna PA, Jankovic J, Vincent A. Comparison of mouse bioassay and immunoprecipitation assay for botulinum toxin antibodies. J Neurol Neurosurg Psychiat 1999;66:612-16. 